\title{
Weighted Gene Correlation Network Analysis (WGCNA) Detected Loss of MAGI2 Promotes Chronic Kidney Disease (CKD) by Podocyte Damage
}

\author{
Zhi Zuo Jian-Xiao Shen ${ }^{\mathrm{b}}$ Yan Panc Juan Pu ${ }^{\mathrm{d}} \quad$ Yong-Gang Li ${ }^{\mathrm{e}}$ \\ Xing-hua Shao ${ }^{c}$ Wan-Peng Wang ${ }^{\mathrm{e}}$ \\ aDepartment of Cardiology, Zhongda Hospital, Medical School of Southeast University, Nanjing \\ City, 'Department of Nephrology, Renji Hospital, School of Medicine, Shanghai Jiaotong University, \\ Shanghai, 'Department of clinical laboratory, Lianshui County People's Hospital, Huai'an City, \\ dDepartment of Radiotherapy, Lianshui County People's Hospital, Huai'an City, eDepartment of \\ Nephrology, Lianshui County People's Hospital, Huai'an City, China
}

\section{Key Words}

Bioinformatics - Weighted gene correlation network analysis (WGCNA) - Chronic kidney disease (CKD) • Podocyte • MAGI2

\begin{abstract}
Background/Aims: Podocyte damage is associated with proteinuria, glomerulosclerosis and decline of renal function. This study aimed to screen critical genes associated with podocyte injury in chronic kidney disease (CKD) by weighted gene correlation network analysis (WGCNA), and explore related functions. Methods: GSE66107, GSE93798, GSE30528, GSE32591 gene expression data including podocyte injury models or glomeruli in CKD patients were downloaded from the GEO database. $R$ was used for data analysis. Differentially expressed genes (DEGs) (FDR $<0.05$ or |Fold Change| $\geq 1.5$ ) in GSE993395 were assessed by WGCNA. According to Gene Ontology (GO) and known podocyte standard genes (PSGs), podocyte injury-associated modules were defined, with hub genes selected based on average intramodular connectivity. The Cytoscape software was used for network visualization. Nephroseq was used to assess the clinical significance of hub genes. Small interfering RNA (siRNA) was used to evaluate the roles of hub genes in podocyte injury Results: Totally 7957 DEGs were screened, with 15 (co.DEGs) altered in all 4 datasets; 4031 DEGs were used for WGCNA, encompassing 12 modules. Green modules (most PSGs and co.DEGs) were significantly enriched in glomerular development, and considered podocyte injury-associated modules. Furthermore, MAGI2 (a hub gene) was also a co.DEG and PSG. Glomerular MAGI2 levels were reduced in various kidney diseases, and positively and negatively associated with glomerular filtration rate and urinary protein levels
\end{abstract}

Z. Zuo, J.-X. Shen and Y. Pan contributed equally to this work.

$\begin{array}{ll}\text { Wan-Peng Wang } & \text { Department of Nephrology, Lianshui County People's Hospital } \\ & \text { Huai'an City, Jiangsu Province (China) } \\ & \text { E-Mail wangwanpeng123@163.com }\end{array}$


in CKD patients. Moreover, MAIG2 knockdown reduced NPHS2, CD2AP and SYNPO levels, and induced podocyte rearrangement and apoptosis. Conclusion: MAGI2 identified by WGCNA regulates cytoskeletal rearrangement in podocytes, with its loss predisposing to proteinuria and CKD.

(C) 2018 The Author(s)

Published by S. Karger AG, Basel

\section{Introduction}

Chronic kidney disease (CKD) is a worldwide public health problem, with increasing prevalence, poor outcomes and high treatment costs [1]. Currently, It is widely accepted that podocyte damage is not only associated with the degree of proteinuria, but also correlates with glomerulosclerosis development and renal function decline [2,3]. Podocyte damage is one of the early-stage events in many human kidney diseases, including minimal change disease (MCD), focal segmental glomerulosclerosis (FSGS), diabetic kidney disease (DKD), membranous nephropathy (MN), lupus nephritis (LN) and other CKDs [4, 5]. The realization that CKD could be reversed by targeting this early-stage event has led to the current theory that novel therapeutics should inhibit podocyte loss. However, the pathogenesis of podocyte loss is complex and not well clarified, and the exact molecular mechanism of podocyte damage in CKD remains largely unexplored.

Traditional molecular biology, which focuses on assessing the functions of single genes or proteins, plays an important role in disease research at the molecular level. However, it is undeniable that traditional molecular biology can only explain the biological system locally, and a more comprehensive exploration of the whole biological system in diseases is very challenging.

Weighted gene correlation network analysis (WGCNA), a powerful method for constructing co-expression networks based on expression data, can reconstruct gene co-expression modules and summarize modules using module eigengenes (ME) and intramodular hub genes [6]. In this work, we utilized a computational strategy to perform a systematic assessment of differentially expressed genes (DEGs) based on 4 datasets, including the expression data from glomeruli of patients with 3 types of CKD (LN, DKD and IgAN) and the podocyte injury model induced by puromycin amino-nucleoside (PAN). Then, DGEs were used for WGCNA based on the GSE99339 dataset including 133 glomerular expression profiles of various CKD patients. WGCNA results combined with DAVID findings and podocyte standard genes (PSGs) defined by previous reports $[7,8]$ were used to identify podocyte injury-associated modules [9], and hub genes were detected by intramodular connectivity. Finally, the Nephroseq online tool (www.nephroseq.org Apr 2017, University of Michigan, Ann Arbor, MI) was used to assess the clinical significance of hub genes, and small interfering RNA (siRNA) was used to explore the role of hub genes in the physiological functions of podocytes.

The present study firstly provides a comprehensive understanding of podocyte injuryassociated genes via a co-expression network, which may guide subsequent experimental studies on podocyte protection in CKD patients.

\section{Materials and Methods}

\section{Microarray data}

The 5 raw gene microarray expression data were downloaded from the GEO database (http:// www.ncbi.nlm.nih.gov/geo/), and included GSE66107, GSE93798, GSE30528, GSE32591 and GSE99339. There were 3 normal podocyte specimens and 3 injured podocyte samples induced by puromycin aminonucleoside (PAN) in the GSE66107 dataset. The remaining samples selected in this study were from the glomerular compartment of the human kidney, as follows. The GSE93798 dataset included samples from 20 IgA nephritis (IgAN) patients and 22 controls; GSE30528 encompassed specimens from 9 diabetic kidney disease (DKD) patients and 13 controls; since we only evaluated genome expression data of the glomeruli 


\section{Cellular Physiology Cell Physiol Biochem 2018;51:244-261 \begin{tabular}{ll|l} 
and BiOChemistry Published onlIne: 16 November 2018 & $\begin{array}{l}\text { (c) } 2018 \text { The Author(s). Published by S. Karger AG, Basel } \\
\text { www.karger.com/cpb }\end{array}$ \\
\hline
\end{tabular}

section, glomeruli samples from the 32 lupus nephritis (LN) and 14 glomeruli control patients were selected for further analysis, which only constituted a part of GSE32591. The GSE99339 dataset included 133 samples based on the GPL19184 platform, which consisted of various types of established CKDs such as LN, IgAN, membranous glomerulonephritis (MGN), hypertensive nephropathy (HT), focal segmental glomerular sclerosis (FSGS), tumorous nephrosis (TN), minimal change nephropathy (MCD), DKD, etc. GSE66107, GSE93798, GSE30528 and GSE32591 data were used for analyzing differential expressed genes (DEGs). Then, DEGs altered in more than one dataset were used in WGCNA to construct a co-expression network based on their normalized expression data in the GSE99339 dataset.

\section{Preprocessing of raw datasets}

Raw expression data were preprocessed with R v3.4.1 (https://www.r-project.org/). The preprocessing of gene expression profile data was performed using the Robust Multi-array Average (RMA) algorithm in affy package of Bioconductor (http://www.bioconductor.org/), including background correction, quantile normalization and pro summarization. To minimize false positives and maintain high expression levels of DEGs for downstream analysis, we only kept the expressed probes with the criterion of "present (P)" in more than $50 \%$ of all samples for each dataset, which was identified by the mas 5 calls function in the affy package [10]. Finally, a total of 26357, 11118, 11296, 7194 and 7114 gene probes were identified in the GSE66107, GSE93798, GSE30528, GSE32591 and GSE99339 datasets for subsequent preprocessing, respectively. As the GSE99339 dataset included several sample batches, the empirical Bayes algorithm (sva package) [11] was used to correct the batch effect of gene expression value in GSE99339. The detailed information for the samples included in this study is summarized in Table 1.

\section{Identification of DEGs}

The Linear Models for Microarray Data package in Bioconductor was applied to identify DEGs by comparing expression values between groups. The corresponding $\mathrm{p}$ values of gene symbols after T-test were defined as adjusted p-values; adjusted p-value $<0.05$ and $\mid$ fold change(FC) $\mid \geq 1.5$ were selected as cut-off criteria for DEGs.

Construction of the gene co-expression network

To explore the interactions between genes, a system biology approach, WGCNA, which converts co-expression measures into connection weight or topology overlap measures, was applied for gene coexpression network construction [12]. The co-expression methodology was typically used for exploring the correlation between gene expression levels. Genes involved in the same pathway or functional compound tend to show similar expression patterns [13]. Therefore, the construction of a gene co-expression network facilitates the identification of genes with similar biological functions [14]. In this work, all DEGs altered in more than one datasets were selected from GSE99339 and entered to construct weighted co-expression modules using the WGCNA package in the R language.

Table 1. Detailed information of the samples included in this study

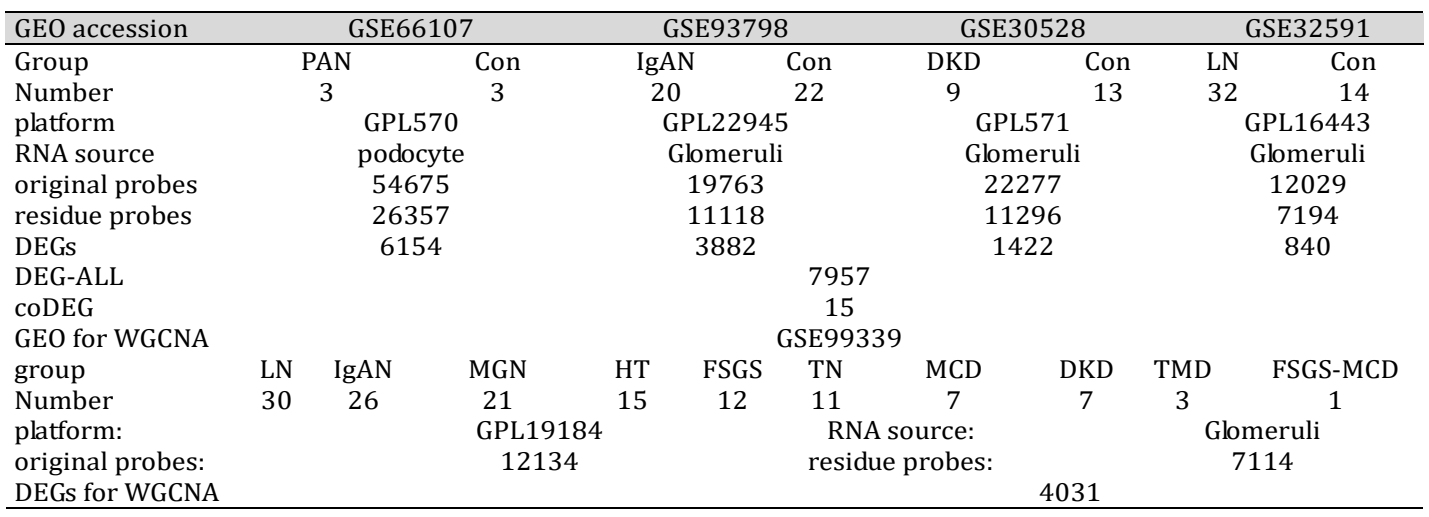




\section{Cellular Physiology Cell Physiol Biochem 2018;51:244-261

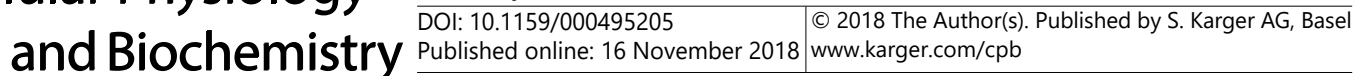 \\ Zuo et al.: Loss of MAGI2 Promoted Podocyte Damage}

\section{Cell Type-Positive Standard Genes in Kidney Disease}

Ju et al. $[7,8]$ have developed an iterative algorithm (in silico Nano dissection), which is a machine learning based approach to statistically define cell type-specific genes using high-throughput data. By applying the algorithm to kidney microarray datasets, it was cross-validated with previously reported data and predicted cell type-positive standard genes for mesangial cells and podocytes in kidney diseases. According to the results, podocyte-positive standard genes (PSGs) included ACTN4, NPHS1, NPHS2, SYNPO, PTPRO, NES, DDN, LRRC7, WT1, TCF21, MAGI1, MAGI2, RAB3A, KIRREL, KIRREL2, KIRREL3, CD2AP, CASK, IQGAP1, TJP1, TRPC6, NCK2, DES, PDPN, PODXL, LMX1B, FAT1, CDH3, PLCE1, CLIC5, CDH13, MME, UCHL1, CD80, Sv2b, PALLD, FYN, PLAUR, DAG1, AGRN, EFNB1, EZR, FOXC2, MAFB, UTRN, MYOC, BASP1, SULF1, SCEL, and CX3CL1.

\section{Enrichment analysis of modules}

DAVID (Database for Annotation, Visualization and Integrated Discovery) (http://david.abcc.ncifcrf. gov/) [15] is a gene functional classification tool that integrates a set of functional annotation tools for investigators to analyze biological functions in massive amounts of genes. GO (Gene Ontology) function and KEGG (Kyoto Encyclopedia of Genes and Genomes) pathway enrichment analyses of all modules were performed based on DAVID.

\section{Podocyte culture and siRNA interference}

The immortalized mouse podocytes MPC5 (provided by Dr. Peter Mundel, Harvard University) have been described in detail previously [16]. Podocytes were maintained in RPMI-1640 (Gibco, USA) supplemented with 5\% FBS. To propagate podocytes, recombinant mouse $\gamma$-IFN $(50 \mathrm{U} / \mathrm{ml}$, R\&D Systems, Minneapolis, MN) was added to the culture medium for incubation at $33^{\circ} \mathrm{C}$. To induce differentiation, podocytes were maintained at $37^{\circ} \mathrm{C}$ for 14 days, and used in experiments. Adriamycin (ADR) is widely used to assess renal diseases by inducing nephrotic syndrome in vivo and podocyte injury in vitro. Therefore, ADR $(0.25 \mu \mathrm{g} / \mathrm{ml}$ TargetMol, USA) was used to cause podocyte injury, by incubation at 0,24 and $36 \mathrm{~h}$, respectively. Then, cells of each group were harvested at $48 \mathrm{~h}$ after differentiation. MAGI2 siRNA and scramble siRNA control were obtained from Applied Biological Materials (ABM) Inc. Transfection was performed with Lipofectamine RNAiMAX reagent (Invitrogen), according to the manufacturer's protocol. Forty-eight hours after transfection, total RNA and protein were extracted. All experiments were performed in triplicate.

\section{Gene and protein expression in podocytes}

Total RNA from podocytes was extracted using TRIzol reagent (TaKaRa Bio, Japan). The gene expression levels of MAGI2, podocin (NPHS2), CD2-associated protein (CD2AP), synaptopodin (SYNPO) were assessed by quantitative real-time reverse transcription polymerase chain reaction (qRT-PCR) using the RT-PCR and SYBR Green kit (TaKaRa Bio, Japan) on a LightCycler 480 Real Time PCR System. The primers used were: MAGI2, 5' - TAAAGACACGACGAACCCCA-3' (forward) and 5'- AAGTTGGGTCTGGGCCTATC' (reverse); NPHS2, $5^{\prime}$ - CCCAGGTACACAGAGCACAA - $3^{\prime}$ (forward) and 5' - CTCACGTCACAACCTTCAG- $3^{\prime}$ (reverse); CD2AP, 5'- AGCTGCTTTCCTAACTCCGT -3' (forward) and 5'- CGCATGGCCTTCTCTTCTTC - $3^{\prime}$ (reverse); SYNPO, 5'AGTGAGGAAGAGGAAGTGCC - $3^{\prime}$ (forward) and $5^{\prime}$ - GAGGCTAGTGTGGTGAGTGT - $3^{\prime}$ (reverse). Conditions for quantitative PCR were $37^{\circ} \mathrm{C}$ for $15 \mathrm{~min}$ and $85^{\circ} \mathrm{C}$ for $5 \mathrm{~s}$ for RT, followed by 40 cycles of $95^{\circ} \mathrm{C}$ for $5 \mathrm{~s}$ and $60^{\circ} \mathrm{C}$ for $30 \mathrm{~s}$. GADPH was used as an endogenous control for data normalization.

Immunoblot was used to assess the protein amounts of MAGI2, NPHS2, CD2AP and SYNPO. Briefly, cells were lysed with RIPA buffer (Thermo Fisher Scientific, USA), and Western blot was performed by standard procedures. The primary antibodies used were goat anti-MAGI2 polyclonal antibody (R\&D Systems, USA), rabbit anti-NPHS2 polyclonal antibody (Proteintech Group, China), rabbit anti-CD2AP polyclonal antibody (Santa Cruz Biotechnology, USA), and rabbit anti-SYNPO polyclonal antibody (Proteintech, CHINA). Antigoat and anti-rabbit horseradish peroxidase-conjugated (HRP) antibodies (Proteintech, China) were used as secondary antibodies. Immunoreactive bands were detected by enhanced chemiluminescence (ECL; Beyotime Biotechnology, China). 


\section{Cellular Physiology Cell Physiol Biochem 2018;51:244-261 \begin{tabular}{ll|l} 
and Biochemistry Published onlIne: I6 November 2018 & $\begin{array}{l}\text { (c) } 2018 \text { The Author(s). Published by S. Karger AG, Basel } \\
\text { www.karger.com/cpb }\end{array}$ \\
\hline
\end{tabular} \\ Zuo et al.: Loss of MAGI2 Promoted Podocyte Damage}

\section{Confocal Fluorescence Microscopy}

On coverslips, podocytes were fixed with $4 \%$ paraformaldehyde, followed by permeabilization with $0.3 \%$ Triton X-100. For F-actin staining, fixed and permeabilized cells were incubated with fluorescein isothiocyanate (FITC) -phalloidin (Yeasen Bio, Shanghai, China). For detecting the NPHS2 protein, after incubation with primary antibody for two hours at room temperature, the slides were incubated with Alexa Fluor ${ }^{8} 647$ Goat Anti-Rabbit IgG (Invitrogen). Analysis was performed by confocal laser-scanning microscopy (Zeiss Lsm510 Meta, Jena, Germany). Photomicrographs of podocytes stained with various antibodies were selected randomly and analyzed in a blinded manner.

\section{Results}

Identification of DEGs in CKD patients and injured podocytes

Based on the above cut-off criteria, a total of 2969 up-regulated and 3185 downregulated DEGs were obtained in injured podocyte samples induced by PAN compared with controls (in GSE66107). Meanwhile, there are 1678 up-regulated and 2204 down-regulated DEGs in glomeruli from IgAN patients compared with controls (in GSE93798). A total of 344 up-regulated and 1078 down-regulated DEGs were identified in DKD patients compared with controls (in GSE30528); 473 up-regulated and 367 down-regulated DEGs were identified in LN patients compared with controls (in GSE30528). As shown in Supplementary Fig. 1A-D (for all supplementary material see www.karger.com/10.1159/000495205), hierarchical cluster analysis of the data revealed that DEGs could be used to distinguish injured podocytes or most CKD samples from respective controls except for three samples included in the GSE93798 dataset (Supplementary Fig. S1A-D). The details of the top 50 up-regulated and 50 down-regulated DEGs in each dataset are provided in Supplementary Table S1-4. The majority of DEGs (87.5\% up-regulated and $82.0 \%$ down-regulated) of PCGs were detected in only one dataset (Supplementary Fig. S1E, F). Meanwhile, only 15 DEGs (4 up-regulated and 11 down-regulated) showed differential expression in all four gene expression profiles, including CDKN1C, MYO5C, C1orf21, KBTBD11, NR3C2, FZD2, MAGI2, LPL, ZFPM2, USP46, PRKAR2B, TRIM38, DESI1, CTSS and ITGB2. Since these 15 DEGs were differently expressed not only in injured podocytes but also in glomeruli of CKD patients, they were considered biochemical makers of CKD, especially in case of podocyte injury.

\section{Construction of weighted gene correlation network analysis (WGCNA)}

Weighted gene correlation network analysis (WGCNA) has been successfully applied in cancer-related studies $[6,17]$. In this study, we applied WGCNA to explore the potential mechanisms of podocyte damage using DEGs obtained in the above analysis. A total of 9217 DEGs explored were altered in more than one datasets, and 1260 DEGs which were overexpressed in one dataset but downregulated in another compared to corresponding controls were removed. Therefore, 7957 DEGs remained for analysis. Consequently, 4031 DEGs found in the processed data of GSE99339 were selected for further investigation. Fig. 1 A shows the cluster of 133 glomeruli samples from various CKD patients, with no abnormal sample. Then, a co-expression network was constructed with the WGCNA package in the $\mathrm{R}$ software. To achieve a scale-free topology $\left(R^{2}>0.9\right)$, we set $\beta=6$ in Fig. $1 B$, then converted the pairwise correlation into an adjacency matrix of connection strengths through the softthresholding approach (connection strength $=\mid$ correlation $\left.\right|^{\beta}$ ). A dissimilarity matrix based on topological overlap measure (TOM) was used to identify gene modules with a dynamic tree-cutting algorithm [6]. All modules were assigned corresponding colors (Fig. 1C). In this study, 4031 DGEs were ultimately categorized into 12 modules with sizes ranging from 52 to 1224 genes. Each module was assigned a unique color: turquoise, blue, brown, yellow, green, red, black, pink, magenta, purple green-yellow and tan represented 1214, 708, 526, 235, $211,195,100,87,70,58,57$ and 52 genes, respectively. Of all genes, 518 were not assigned to any of the 12 modules, and designated as grey (Table 2, Fig. 1C). A complete list of genes in each module is shown in Supplementary Table S5.

\section{KARGER}


Fig. 1. Weighted gene correlation network analysis (WGCNA). (A) Cluster results and traits of data samples. The color intensity was proportional to age, height, weight, Body Mass Index (BMI), Blood Urea Nitrogen (BUN), Serum creatinine (Scr), CKD stage, e $\mathrm{st}$ i $\mathrm{m}$ a t e d glomerular filtration rate (eGFR), mean blood pressure (BP). For gender, white and red indicate male and female, respectively. For disease status (DKD, MCD, TMD, TN, HT, FSGS-MCD, FSGS, IgAN, MGN and LN), white and red indicate "NO" and "YES", respectively. Missing data are represented in grey. Determination of parameter $\beta$ of the adjacency function in the WGCNA algorithm. The left panel shows the scale-free

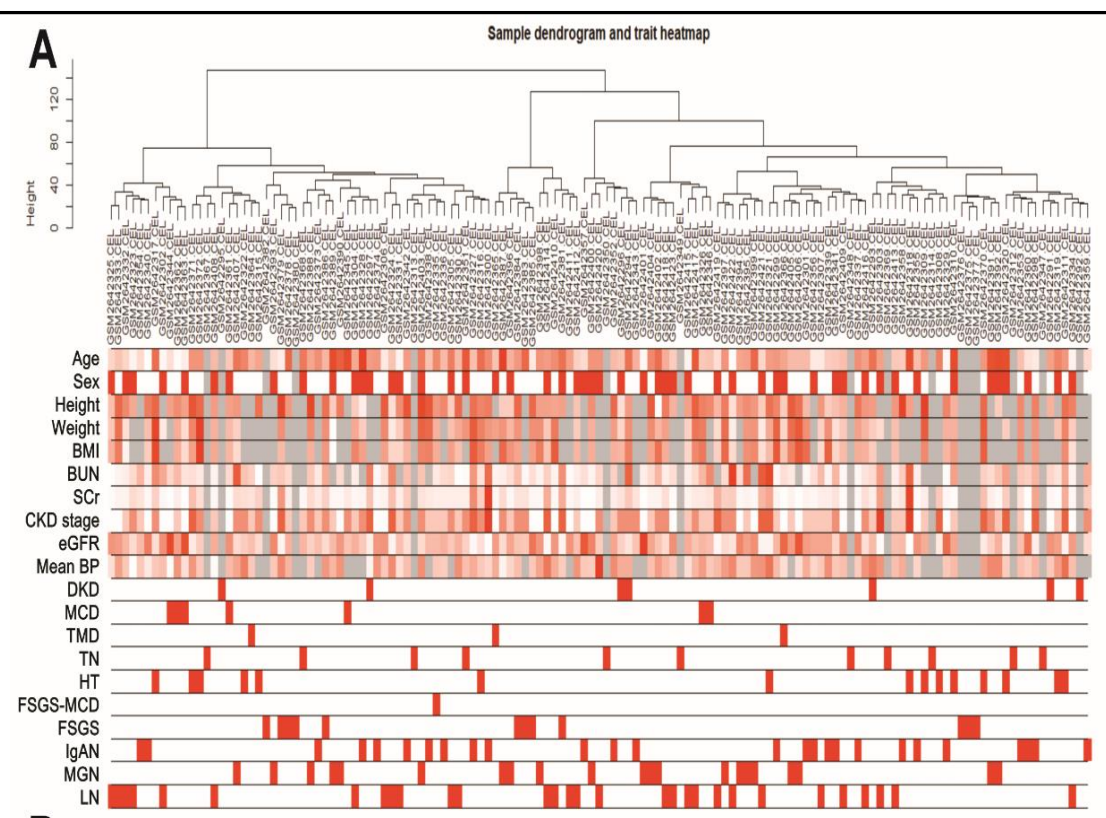
B
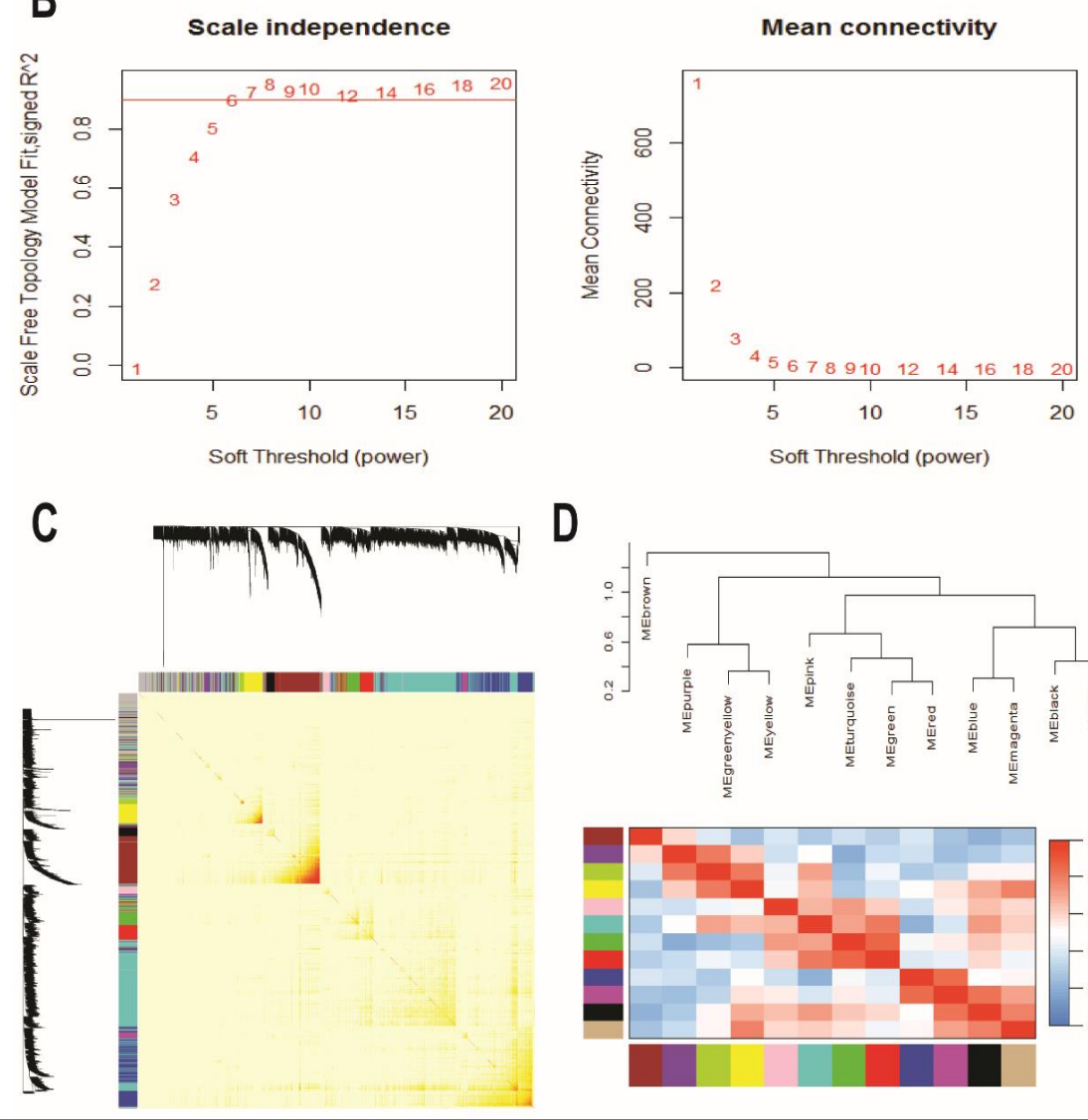

D
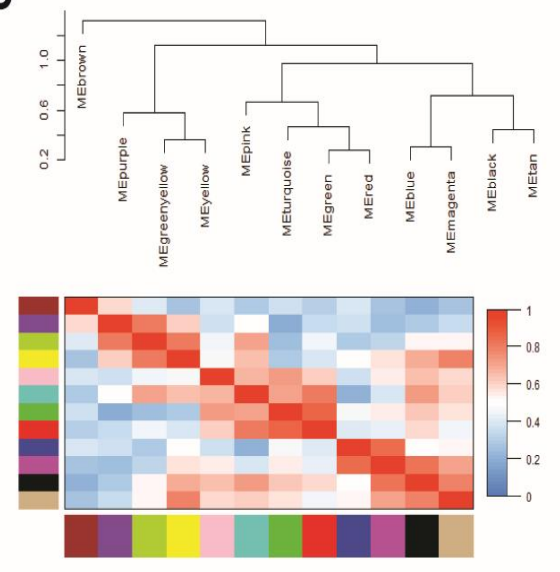
topology fitting index $\left(\mathrm{R}^{2}, \mathrm{y}\right.$-axis) as a function of the soft-thresholding power ( $\mathrm{x}$-axis). The right panel displays the mean connectivity (degree, $y$-axis) as a function of the soft-thresholding power (x-axis). Red Arabic numbers in the panels denote different soft-thresholds. The red line in the left panel indicates $\mathrm{R}^{2}=0.9$. There is a trade-off between maximizing the scale-free topology model fitting index $\left(\mathrm{R}^{2}\right)$ and maintaining a high mean number of connections. Thus, we set $\beta=6$. (C) Construction of the gene correlation network. Each color represents a certain gene module. (D) Clustering tree based on modules' eigengenes. 


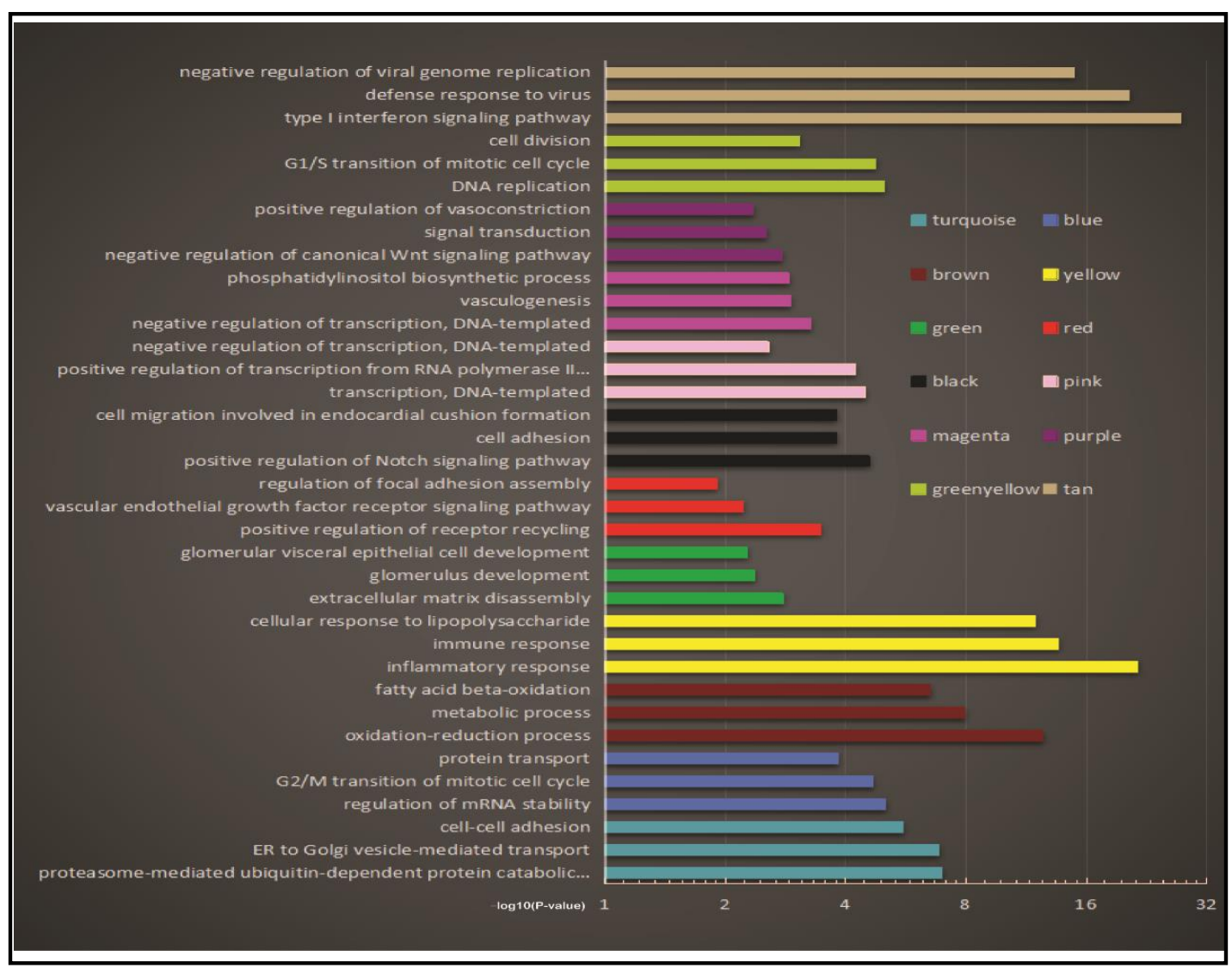

Fig. 2. Gene ontology (GO) and KEGG analyses. (A) Bar plot of representative GO biological process (BP) terms of the 12 modules. Tree representative GO BP terms were selected in each module. The y-axis depicts names of BP terms, and the $\mathrm{x}$-axis represents - $\log 10$ (P-value). Bar color denotes the module color.

Module-based functional characterization of DEGs with co-expression network analysis

Module eigengenes (ME) were used to represent each module, which was determined by the first principal component. First, we analyzed the relationship between the modules and pathological parameters by calculating the correlation value of ME for each module (Supplementary Figure S2). The results showed no specific link between single modules and serum creatinine (Scr) or estimated glomerular filtration rate (eGFR). This could be because of the small sample size, and altered gene expression in glomerular damage is an early stage event in CKD, while pathological changes of cardinal features such as tubular cell atrophy and interstitial injury, as common cardinal features in CKD, mainly lead to decreased eGFR. Meanwhile, several modules were significantly associated with certain clinic parameters, e.g. the turquoise $(\mathrm{P}=0.045)$ module, which was negatively associated with body mass index (BMI), while significant positive correlation was found between the yellow module and mean blood pressure $(\mathrm{P}=0.008)$.

To further determine the biological functions of these genes in the 12 modules, DAVID[9] was used to mine the modules' biological significance, including GO biological process (BP) terms and KEGG pathways. Supplementary Table S6 lists the top 30 significantly enriched GO BP terms $(\mathrm{p}<0.05)$ for each module. Fig. 2 displays three representative terms for each module. Supplementary Table S7 lists complete KEGG pathway terms $(\mathrm{p}<0.05)$ for each module. Table 3 lists the top three KEGG pathways with $\mathrm{p}<0.05$ for each module. According to major biological processes and the number of PSGs included in various modules, the latter were roughly parceled into two sections, including podocyte-specific and nonspecific injury associated modules, as follows. 


\section{Cellular Physiology Cell Physiol Biochem 2018;51:244-261 \begin{tabular}{ll|l} 
and Biochemistry Published onlıne: 16 November 2018 & $\begin{array}{l}\text { (c) } 2018 \text { The Author(s). Published by S. Karger AG, Basel } \\
\text { www.karger.com/cpb }\end{array}$ \\
\hline
\end{tabular} \\ Zuo et al.: Loss of MAGI2 Promoted Podocyte Damage}

Table 2. Overview of 12 modules constructed by WGCNA. (PSG, podocyte standard gene; co.DEG, genes altered in all 4 datasets; hub, the $5 \%$ of genes with highest connectivity were defined as hub genes in each module; co.DEG-hub, intersection of co.DEG and hub; PSG -hub, intersection of PSG and hub)

\begin{tabular}{|c|c|c|c|c|c|c|}
\hline Medule & $\mathrm{N}$ & PSG $(N=22)$ & co.DEG $(\mathrm{N}=15)$ & hub & co.DEG-hub & PSG -hub \\
\hline turquoise & 1214 & IQGAP1 PALLD & - & 61 & - & - \\
\hline blue & 708 & CASK AGRN & FZD2 ZFPM2 DESI1 & 36 & - & - \\
\hline brown & 526 & - & - & 27 & - & - \\
\hline grey & 518 & - & LPL & 26 & - & - \\
\hline yellow & 235 & PLAUR BASP1 & MY05C TRIM38 ITGB2 CTSS & 12 & ITGB2 CTSS & - \\
\hline green & 211 & TJP1 FYN MME PLCE1 MAGI2 TCF21 NES CLIC5 NPHS2 DDN & CDKN1C KBTBD11 C1orf21 MAGI2 PRKAR2B NR3C2 & 11 & MAGI2 & MAGI2 PLCE1 CLIC5 MME \\
\hline red & 195 & FAT1 SULF1 CD2AP UCHL1 & USP 46 & 10 & - & SULF1 \\
\hline black & 100 & $\mathrm{CDH} 13$ & - & 5 & $\cdot$ & - \\
\hline pink & 87 & - & - & 5 & - & - \\
\hline magenta & 70 & CX3CL1 & - & 4 & - & - \\
\hline purple & 58 & - & - & 3 & - & - \\
\hline greenyellow & 57 & - & - & 3 & - & - \\
\hline $\tan$ & 52 & & & 3 & & \\
\hline
\end{tabular}

Table 3. Top three KEGG pathways with $\mathrm{p}<0.05$ in each module (Count, number of Genes involved in a given term; \%, Percentage (involved genes/total genes); P-Value, Modified Fisher Exact P-value)

\begin{tabular}{|c|c|c|c|c|}
\hline Module & Term & Count & $\%$ & P-Value \\
\hline \multirow[t]{3}{*}{ turquoise } & hsa04141:Protein processing in endoplasmic reticulum & 32 & 2.68 & $9.31 \mathrm{E}-07$ \\
\hline & hsa04120:Ubiquitin mediated proteolysis & 27 & 2.26 & $3.71 \mathrm{E}-06$ \\
\hline & hsa03015:mRNA surveillance pathway & 18 & 1.51 & 2.19E-04 \\
\hline \multirow[t]{3}{*}{ blue } & hsa03050:Proteasome & 9 & 0.01 & 3.77E-04 \\
\hline & hsa04141:Protein processing in endoplasmic reticulum & 17 & 0.02 & $1.62 \mathrm{E}-03$ \\
\hline & hsa05221:Acute myeloid leukemia & 9 & 0.01 & $1.97 \mathrm{E}-03$ \\
\hline \multirow[t]{3}{*}{ brown } & hsa01100:Metabolic pathways & 116 & 0.14 & $1.15 \mathrm{E}-22$ \\
\hline & hsa01200:Carbon metabolism & 27 & 0.03 & 8.57E-14 \\
\hline & hsa01130:Biosynthesis of antibiotics & 34 & 0.04 & $4.50 \mathrm{E}-12$ \\
\hline \multirow[t]{3}{*}{ yellow } & hsa04064:NF-kappa B signaling pathway & 15 & 0.04 & $2.46 \mathrm{E}-10$ \\
\hline & hsa05152:Tuberculosis & 18 & 0.05 & $1.01 \mathrm{E}-08$ \\
\hline & hsa04060:Cytokine-cytokine receptor interaction & 20 & 0.06 & $1.55 \mathrm{E}-08$ \\
\hline \multirow[t]{3}{*}{ green } & hsa05205:Proteoglycans in cancer & 8 & 0.02 & $1.06 \mathrm{E}-02$ \\
\hline & hsa04520:Adherens junction & 5 & 0.02 & $1.06 \mathrm{E}-02$ \\
\hline & hsa04015:Rap1 signaling pathway & 8 & 0.02 & $1.36 \mathrm{E}-02$ \\
\hline \multirow[t]{3}{*}{ red } & hsa00563:Glycosylphosphatidylinositol(GPI)-anchor biosynthesis & 4 & 0.01 & $2.57 \mathrm{E}-03$ \\
\hline & hsa04142:Lysosome & 6 & 0.02 & $1.10 \mathrm{E}-02$ \\
\hline & hsa04141:Protein processing in endoplasmic reticulum & 7 & 0.02 & $1.11 \mathrm{E}-02$ \\
\hline \multirow[t]{3}{*}{ black } & hsa04330:Notch signaling pathway & 4 & 0.03 & $3.20 \mathrm{E}-03$ \\
\hline & hsa04514:Cell adhesion molecules (CAMs) & 5 & 0.03 & $1.13 \mathrm{E}-02$ \\
\hline & hsa04270:Vascular smooth muscle contraction & 4 & 0.03 & 3.73E-02 \\
\hline pink & hsa04919:Thyroid hormone signaling pathway & 3 & 0.02 & $3.84 \mathrm{E}-02$ \\
\hline \multirow[t]{3}{*}{ magenta } & hsa04360:Axon guidance & 6 & 0.06 & 5.98E-04 \\
\hline & hsa04070:Phosphatidylinositol signaling system & 5 & 0.05 & $1.94 \mathrm{E}-03$ \\
\hline & hsa00562:Inositol phosphate metabolism & 4 & 0.04 & $6.78 \mathrm{E}-03$ \\
\hline \multirow[t]{3}{*}{ purple } & hsa04510:Focal adhesion & 6 & 0.06 & $1.06 \mathrm{E}-03$ \\
\hline & hsa05202:Transcriptional misregulation in cancer & 5 & 0.05 & $3.82 \mathrm{E}-03$ \\
\hline & hsa05205:Proteoglycans in cancer & 5 & 0.05 & $7.09 \mathrm{E}-03$ \\
\hline \multirow[t]{3}{*}{ greenyellow } & hsa04110:Cell cycle & 7 & 0.07 & $1.23 \mathrm{E}-05$ \\
\hline & hsa03030:DNA replication & 4 & 0.04 & $4.79 \mathrm{E}-04$ \\
\hline & hsa04115:p53 signaling pathway & 4 & 0.04 & 2.93E-03 \\
\hline \multirow[t]{3}{*}{$\tan$} & hsa05164:Influenza A & 9 & 0.16 & $2.12 \mathrm{E}-08$ \\
\hline & hsa05168:Herpes simplex infection & 9 & 0.16 & $3.15 E-08$ \\
\hline & hsa05162:Measles & 7 & 0.13 & $1.94 \mathrm{E}-06$ \\
\hline
\end{tabular}

\section{Podocyte-nonspecific injury associated modules}

For instance, the yellow and brown modules. The yellow module, which contained 251 genes and only two PCGs, and was positively associated with blood pressure $(r=0.23, P=0.008$, Supplementary Figure S2), showed functional enrichment in inflammatory response, 


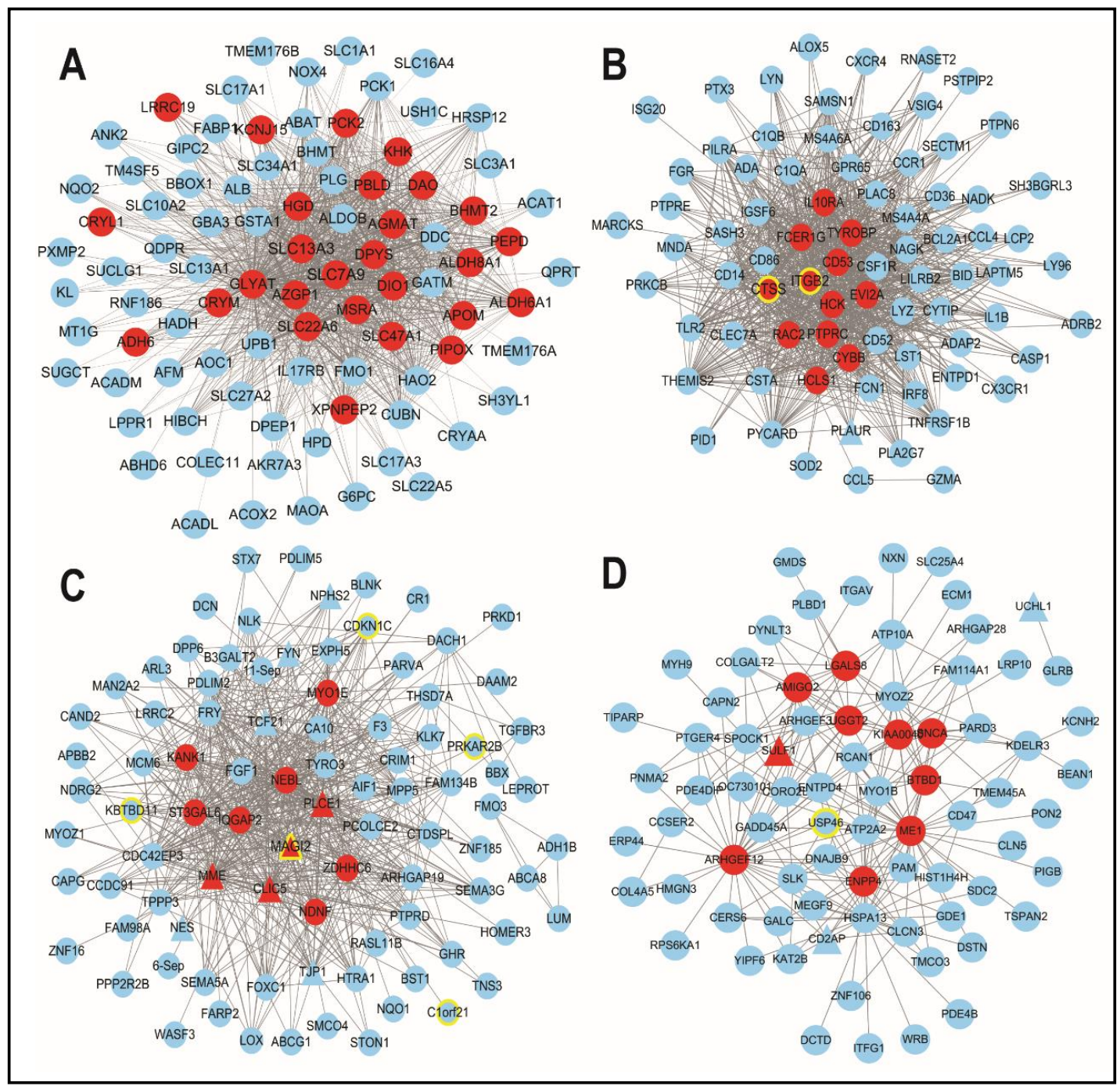

Fig. 3. Cytoscape network visualization of genes in the brown (A), yellow (B), green (C) and red (D) modules, in which only edges with weight $(\mathrm{w}$ ) above a threshold of $0.3,0.3,0.15,0.05$ and 0.05 are displayed, respectively. The red nodes denote hub genes for each module; the triangular nodes denote podocyte standard genes (PSGs); the nodes with yellow border represent genes altered in all 4 datasets; the $5 \%$ of genes with the highest connectivity was defined as hub genes (co.DEGs) (hub).

immune response, cellular response to lipopolysaccharide and apoptotic process, and so on (Fig. 2, Supplementary Table S6). Pathway analysis further revealed that genes in this module were enriched in signal transduction pathways such as NF-kappa B [18], TNF[19],Toll-like receptor [20] and NOD-like receptor [21] signaling pathways (Table 3, Supplementary Table S7). These GO terms or pathways were shown to be associated with podocyte damage and blood pressure regulation [22, 23] in multiple reports. However, these pathways are not podocyte-specific, and also involved in the functions of other kidney cells, including mesangial [24] and endothelial [25] cells. Similar observations were made for the brown module, which was composed of 533 genes and no PSG, and negatively correlated with blood pressure ( $r=-0.2, \mathrm{P}=0.021$, Supplementary Figure S2). The BP terms of the brown module were associated with the functions of oxidation-reduction, metabolic process and fatty acid beta-oxidation, and their disorders were revealed to be connected with a predisposition to cell injury (Fig. 2, Supplementary Table S6). In addition, pathway analysis showed genes in the brown module were mainly enriched in Metabolic pathways, Carbon metabolism, and

\section{KARGER}




\section{Cellular Physiology Cell Physiol Biochem 2018;51:244-261 \begin{tabular}{l|l|l} 
and Biochemistry Published onlIne: I6 November 2018 & $\begin{array}{l}\text { (c) } 2018 \text { The Author(s). Published by S. Karger AG, Basel } \\
\text { www.karger.com/cpb }\end{array}$ \\
\hline
\end{tabular} \\ Zuo et al.: Loss of MAGI2 Promoted Podocyte Damage}

Glyoxylate and dicarboxylate metabolism, which are also associated with the regulation of cell metabolism (Table 3, Supplementary Table S7). Therefore, it is reasonable to infer that genes in the yellow and brown modules may play roles in maintaining survival of not only podocytes, but also glomerular endothelial cells and mesangial cells.

\section{Podocyte-specific injury modules}

According to major biological processes and KEGG pathway analysis, the green and red modules stood out. There were 211 DEGs in the green module, including 10 PSGs and 6 co.DEGs (Table 2), most than in the other modules. as shown in Fig. 2 and Supplementary Table S6, genes in the green module were significantly enriched in processes such as extracellular matrix disassembly, glomerulus development, and glomerular visceral epithelial cell development. Significant KEGG pathways included several signaling pathways relevant to podocyte function such as Adherens junction and the Rap1 signaling pathway (Table 3, Supplementary Table S7). The results were confirmed and visualized in a functionally grouped network created by the Cytoscape software v3.5.1 combined with ClueGO plug-in (v2.3.5) (Supplementary Fig. 3). Similarly, 211 genes in the red module, which contained 4 PSGs and 1 co.DEGs, also showed significant enrichment in functions involved in response to endoplasmic reticulum stress, cellular response to mechanical stimulus and regulation of focal adhesion assembly. Most of these GO terms in the red and green modules have been reported to associate with podocyte damage in CKD patients.

To explore the relationship between modules, clustering analysis for ME was applied. The results showed the green and red modules were very close, suggesting that genes in the red and green modules played similar roles in maintaining podocyte function. Especially, the green module included more PSGs and co.DEGs than other modules, so we suspect that genes included in the green module were more likely to specifically participate in triggering the injury mechanism of podocytes compared with those of other modules. Therefore, the green module was defined as podocyte-injury associated module in the present study.

\section{Hub-based analysis}

Highly connected hub nodes are central to the network's architecture [6] and studies suggested that genes more centralized in the network are more likely to be key drivers to respective cellular functions compared with peripheral genes [26]. Therefore, more important nodes can be obtained by identifying hub nodes. In this study, the top $5 \%$ of nodes with highest connectivity were defined as hub genes in each module [27]. Fig. 3A-D are the networks of hub genes in the brown, yellow, green and red modules, only displaying connections with weight $(\mathrm{w})$ above threshold values of $0.3,0.15,0.05$, and 0.05 , respectively. Clearly, hub genes (red nodes) exhibited high connectivity with neighboring genes whose functions are consistent with GO and KEGG findings. Taking the brown module as an example, which showed functional enrichment in metabolic and oxidation-reduction processes, some hub genes have been also reported to participate in similar processes, e.g. the SLC13 Family (SLC13A3) [28, 29], SLC22 Family (SLC22A6) [30] or MSRA that plays a protective role in the progression of UUO-induced kidney fibrosis via suppression of fibrotic responses caused by oxidative stress [31]. Similarly, some hub genes in the yellow module have been identified as pro-inflammatory factors in a variety of human diseases, including CTSS and ITGB2 [32], consistent with the BP term or KEGG pathway enriched in the yellow module. Meanwhile, some well-known inflammatory factors also had high connectivity to these hub genes, e.g. IL1B, CXCR4 and CASP1. These observations further supported the notion that proposed roles for hub genes of unknown functions may be inferred from clusters of genes similarly expressed across many biological conditions.

For the red module, one of the hub genes was ARHGEF12, which was highly connected to the well-known podocyte-associated gene CD2AP, which was reported to be enriched in glomerular expression and necessary for normal glomerular filtration barrier function by spatiotemporal regulation of RhoA activity [33, 34]. The most important module was the green, which was defined as podocyte injury-associated module according to the above 


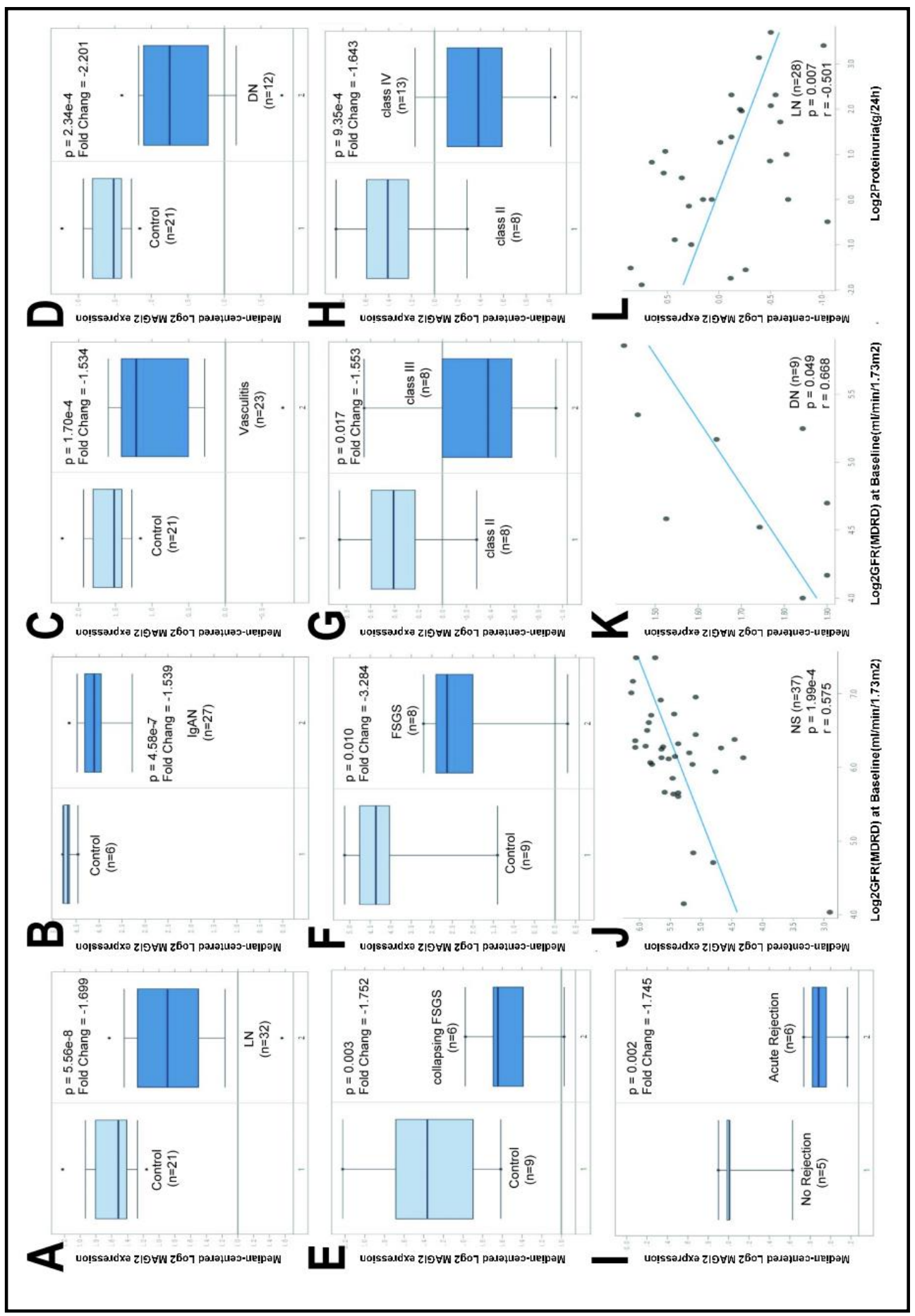

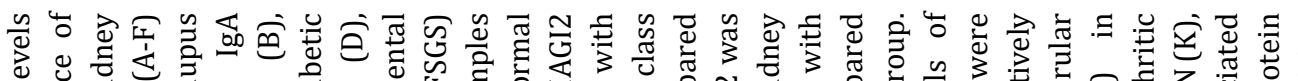

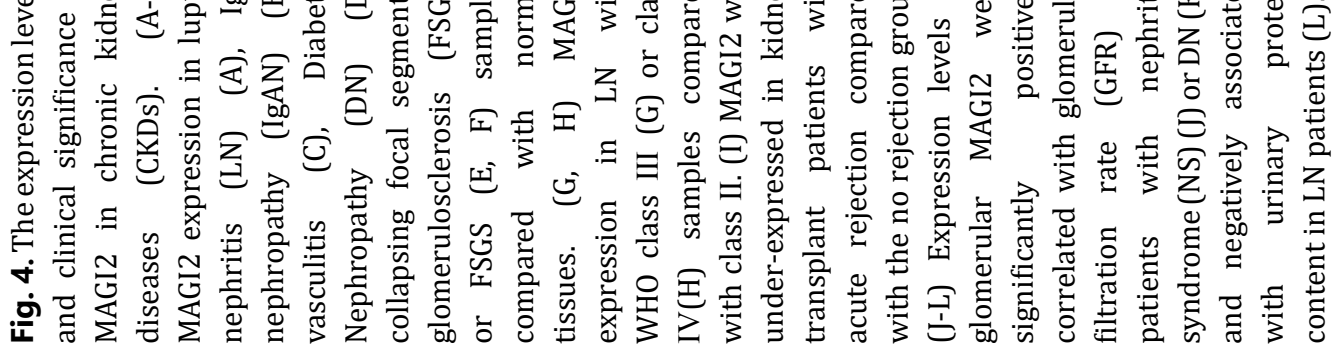
KARGER 


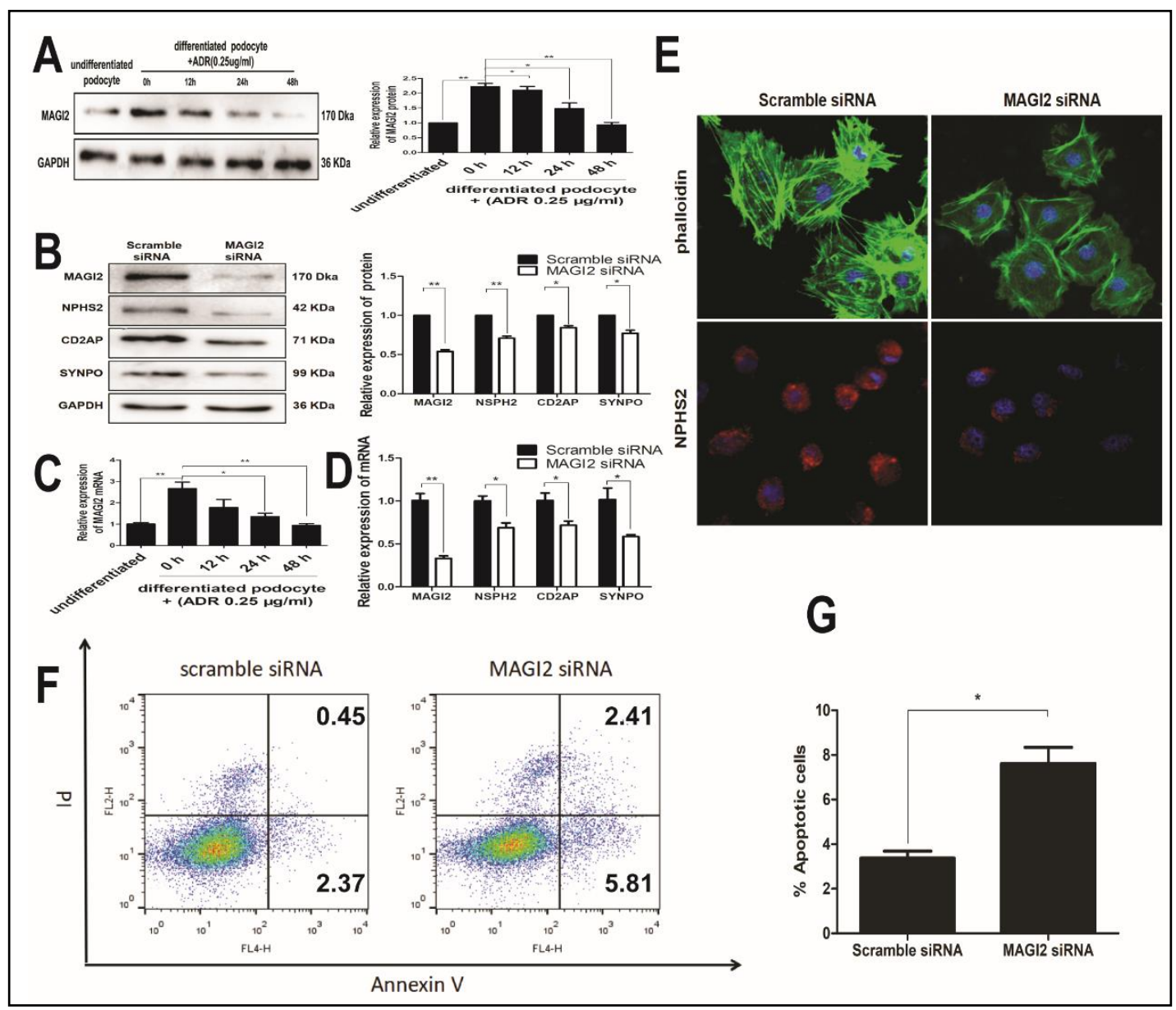

Fig. 5. MAGI2 regulates cytoskeleton rearrangement in mouse podocyte cultures. $(A, C)$ Immunoblotting (A, left: western blot bands; right: relative quantitative analysis) and real-time PCR (C) were used to assess the protein and mRNA expression levels of MAIG2 in undifferentiated podocytes or injured podocytes induced by Adriamycin (ADR, 0.25 $\mu \mathrm{g} / \mathrm{ml}$ ) (n); (B, D) Decreased expression levels of MAGI2, NPHS2, CD2AP and SYNPO as assessed by immunoblot (B, left: western blot bands; right: relative quantitative analysis) and real-time PCR (D) after transfection with MAGI2 siRNA (50 nM for 48h). (E) Upper panel, F-actin was visualized with FITC-conjugated phalloidin; lower panel, expression of the MAGI2 protein was confirmed by Laser Scanning Confocal Microscopy. (F) Podocyte apoptosis after transfection with MAGI2 siRNA (50 nM for 48h) was determined by flow cytometry. (G) Quantitative analysis of Annexin V+ PI- and Annexin V+ PI+ podocytes. Data are mean \pm SEM, $n=3$ per group. $* \mathrm{p}<0.05,{ }^{* *} \mathrm{p}<0.01$.

analysis. As shown in Fig. 3C, it included more PSGs and co.DEGs compared with all other modules. In this module, 11 hub genes exhibited high connectivity with neighboring genes (FGF1, AIF1, CR1, NPSH2, PLCE1, etc.), whose functions are involved in stabilizing the intracellular environment and maintaining podocyte survival by balancing anabolic metabolism and catabolism. Specifically, NPSH2, a podocyte-specific gene that encodes a protein that plays a role in regulating glomerular permeability was found; mutations in this gene cause steroid-resistant nephrotic syndrome [35]. MAGI2, one of the hub genes in the green module, particularly attracted our attention; it was also defined as one of the PSGs and significantly downregulated in injured podocytes and the glomeruli of IgA, DKD or LN patients by gene expression data analysis. This strongly suggested that MAGI2 not only could be well suited as a CKD biomarker, especially in case of podocyte damage, but also contributed to the process of occurrence and development of podocyte damage. 


\section{Cellular Physiology Cell Physiol Biochem 2018;51:244-261 \begin{tabular}{ll|l} 
and Biochemistry & $\begin{array}{l}\text { DOl: 10.1159/000495205 } \\
\text { Publisned online: 16 November } 2018\end{array}$ & $\begin{array}{l}\text { (2018 The Author(s). Published by S. Karger AG, Basel } \\
\text { www.karger.com/cpb }\end{array}$ \\
\cline { 2 - 3 }
\end{tabular} \\ Zuo et al.: Loss of MAGI2 Promoted Podocyte Damage}

\section{Reduced expression of MAGI2 is associated with CKD progression}

To further explore the relationship between CKD and MAGI2 in patients, Nephroseq (www.nephroseq.org Apr 2018, University of Michigan, Ann Arbor, MI) data were analyzed in various CKD patients. As shown in Fig. 4A-F, the expression levels of MAGI2 were reduced in the glomeruli of LN (Fig. 4A), IgAN (Fig. 4B) and DN (also referred to as DKD, as above) (Fig. 4D) patients, in agreement with the above expression profile data, as well as in vasculitis (Fig. 4C) and focal segmental glomerulosclerosis (FSGS) samples (Fig. 4E, F) compared with normal controls. Meanwhile, the expression levels of MAGI2 were decreased in LN patients with WHO class III (Fig. 4G) and IV (Fig. 4H), compared with WHO class II cases. In addition, MAGI2 was downregulated in kidney transplant patients with acute rejection compared with cases without rejection (Fig. 4i). What's more, the expression levels of glomerular MAGI2 were significantly positively correlated with the glomerular filtration rate (GFR) in patients with nephritic syndrome (NS) (Fig. 4J) or diabetic nephropathy (DN) (Fig. 4K), and negatively associated with urinary protein content in LN patients (Fig. 4L). These results suggested that reduced expression of glomerular MAGI2 may participate in the pathogenesis of kidney damage and CKD progression.

\section{Loss of MAGI2 promotes podocyte cytoskeletal rearrangement}

To confirm the role of the MAGI2 gene in the pathogenesis of podocyte damage, conditionally immortalized transgenic mouse podocytes (MPC5) were used. These cells grow into a cobblestone morphology at the permissive temperature of $33^{\circ} \mathrm{C}$ and are maintained at $37^{\circ} \mathrm{C}$ for 14 days before experimentation to induce differentiation. First, we assessed MAGI2 expression in undifferentiated podocytes or injured podocytes induced by Adriamycin (ADR), which was often used in nephropathic models. As shown in Fig. 5A and C, MAGI2 protein and mRNA levels were increased in differentiated podocytes compared with the undifferentiated counterparts. Meanwhile, in differentiated podocytes, exposure to ADR caused a substantial loss of MAGI2 at the mRNA and protein levels (Fig. 5A and C). To determine if MAGI2 influences cytoskeletal remodeling in podocytes, as an early event in CKD development that drives a shortening and simplification of podocyte foot processes, we knocked down MAGI2 in differentiated podocytes by siRNA interference. Western blot and real-time PCR confirmed the knockdown efficiency of MAGI2 siRNA (50 nM) at 48h. In addition to MAGI2 reduction, the mRNA and protein levels of NSPH2, SYNPO and CD2AP, which have fundamental functions in basic biochemical processes in podocytes [36] (Fig. 5B and D), were also decreased after MAGI2 silencing. Similar results of down-regulated NSPH2 are shown in Fig. 5E, which depicts confirmation by immunofluorescence. In addition, F-actin had overtly recovered arrangement by confocal fluorescence microscopy and FITC-phalloidin staining. In the scramble siRNA group, F-actin was characterized by highly ordered, parallel, contractile actin filament bundles, and disorganized, with reorganized, short, branched actin filaments filled in the cytoplasm of podocytes treated with MAGI2 siRNA $50 \mathrm{nM}$ for $48 \mathrm{~h}$ (Fig. 5E). What's more, flow-cytometric analysis showed that apoptosis was increased after MAGI2 silencing (Fig. 5F and G). These results suggested that MAGI2 inhibition promoted cytoskeletal rearrangement and injury in podocyte cultures.

\section{Discussion}

As a global health problem, CKD affects $10-16 \%$ of the adult population in Asia, Europe, and the USA [37-39], and may further develop into kidney failure. Over the past ten years, accumulating evidence indicates that proteinuria is associated with renal function decline, and severe proteinuria can individually predict all-cause mortality in CKD patients, independent of numerous confounding factors [40]. It is widely accepted that effective interventions to reduce proteinuria in CKD patients should constitute the top priority for future studies [41]. Although emerging evidence suggests that podocytes serve as the final barrier to urinary protein loss through the maintenance of foot-processes and an interposed 
slit-diaphragm (SD) [42], current understanding of the mechanism of podocyte injury is very limited and remains to be elucidated.

Gene expression analysis by microarrays has become one of the most widely used high-throughput methods for gathering genome-wide functional data. In this study, we first analyzed differential gene expression in four datasets, including typical podocyte injury models induced by PAN and microarray data of glomeruli from three common CKD types, to provide insights into the possible mechanisms of podocyte damage and facilitate the early prediction of podocyte injury in CKD patients. As a result, 15 DEGs (co.DEGs) were altered significantly in the four datasets, including CDKN1C, MYO5C, C1orf21, KBTBD11, NR3C2, FZD2, MAGI2, LPL, ZFPM2, USP46, PRKAR2B, TRIM38, DESI1, CTSS and ITGB2. Identification of these dysregulated genes helps predict patient outcomes and guides treatments. Meanwhile, some of these genes have been previously reported. For example, CDKN1C, also known as P57, is very important in cell cycle regulation, and cells deficient in this gene exhibit high rates of cell growth and decreased differentiation, and CDKN1C/p57 is down-regulated in many proliferation diseases, including Beckwith-Wiedemann Syndrome and autosomal dominant polycystic kidney disease (ADPKD) $[43,44]$. In addition, previous findings reveal that damaged podocytes may inhibit p57 protein expression, but activate a cyclin D1independent cell-cycle mechanism to promote glomerular epithelial cell (GEC) proliferation in the cellular lesions of FSGS [45]. The NR3C2 gene encodes the mineralocorticoid receptor that mediates aldosterone effects on salt and water balance within restricted target cells. Mutations in NR3C2 cause autosomal dominant pseudo hypoaldosteronism type I [46], a disorder characterized by urinary salt wasting. A recent study showed that NR3C2 is downregulated in stable and chronic rejection (CR) patients compared with kidneytransplant recipients with "operational tolerance" [47]. CTSS, also known as cathepsin S, is a lysosomal cysteine proteinase that may participate in the degradation of antigenic proteins to peptides for presentation on MHC class II molecules; in addition, circulating cathepsin S levels are associated with GFR decline in mice and humans [48], and specific inhibition of cathepsin S could diminish the effects of cardiovascular disease (CVD), especially in patients with CKD [49]. Still, only few co.DEGs have been reported in association with CKD to date, especially for their roles in podocyte damage. Thus, the unreported co.DEGs could provide helpful information for possible biomarkers in further studies.

It is well accepted that co-expressed genes are more likely to be co-regulated and functionally related [50]. Therefore, identifying co-expressed protein-coding genes can help assign the functions of disease-causing gene [51]. This has been successfully applied to assess protein-coding genes, e.g. distinguishing dysfunctional regulatory subnetworks and identifying candidate biomarkers in multiple diseases [52]. However, few studies have used this tool to assess genes related to podocyte damage. The objectives of the current study were to gain molecular insights into CKD progression and identify candidate gene groups involved in podocyte damage. Finally, we obtained 12 modules with sizes ranging from 52 to 1,214 genes. Next, we revealed that the green module containing 211 DEGs likely played key roles in the multistep development of podocyte damage, covering a wide range of functions in glomerulus development, extracellular matrix disassembly, and glomerular visceral epithelial cell development, and also comprised more co.DEGs and PSGs compared with the other modules. Meanwhile, multiple genes in the green module have been reported to be involved directly in podocyte lesion, e.g. MME [53], CR1 [54], NPHS2 and IQGAP2 [55]. These results imply that genes in the green module may play important roles in multiple CKDs, particularly participating in podocyte damage. Therefore, it is reasonable to define the green module as a podocyte injury-associated module; this provides a comprehensive analysis of podocyte injury-associated genes based on the co-expression network, and may guide subsequent experimental studies on podocyte protection.

It should be noted that membrane associated guanylate kinase, WW and PDZ domain containing 2 (MAGI2) was differentially expressed in all 4 datasets, and also defined as the one of hub genes in the green module according to average connectivity. MAGI2 was also one of the PSGs, also known as AIP1 or SSCAM. Its encoded protein is characterized by two 


\section{Cellular Physiology Cell Physiol Biochem 2018;51:244-261 \begin{tabular}{ll|l} 
and Biochemistry & $\begin{array}{l}\text { DOl: 10.1159/000495205 } \\
\text { Publisned online: 16 November } 2018\end{array}$ & $\begin{array}{l}\text { (2018 The Author(s). Published by S. Karger AG, Basel } \\
\text { www.karger.com/cpb }\end{array}$ \\
\cline { 2 - 3 }
\end{tabular} \\ Zuo et al.: Loss of MAGI2 Promoted Podocyte Damage}

WW domains, a guanylate kinase-like domain, and multiple PDZ domains, with structural similarity to the membrane-associated guanylate kinase homologue (MAGUK) family. As a tight junction protein in epithelial tissues, MAGI2 has a critical role in maintaining the functional structure of the slit diaphragm, and is essential in the functioning of the kidney filtration barrier [56]; as one of the WT1 target genes, it is required for podocyte development [57]. More importantly, MAGI2 null mice show progressive proteinuria as early as 2 weeks postnatally [58]. In the present study, glomerular MAGI2 expression and its clinical implication were examined in many types of CKD using Nephroseq data, and the results showed significantly downregulated MAGI2 in CKDs, corroborating previous findings. In addition, there were negative and positive associations of MAGI2 expression with proteinuria and eGFR, respectively. Podocytes express several specific cytoskeletal proteins, including the slit diaphragm (SD)-localized protein NPHS2 (podocin) and actinassociated proteins SYNPO (synaptopodin) and CD2AP, which participate in the maintenance of slit diaphragm and foot process integrity [59-61]. Finally, we used MAGI2 knockdown in podocytes to examine how this gene affects glomerular function and CKD progression; the results indicated that MAGI2 silencing in podocytes led to significantly reduced expression levels of NPHS2, CD2AP and SYNPO, and caused distinct cytoskeletal rearrangement, which is widely considered an early event in the development of CKD. This observation could at least in part explain the clinical significance of MAGI2 in CKD, as mentioned above. These results imply that the MAGI2 gene may constitute a candidate biomarker in CKD diagnosis and serve as a therapeutic gene target for podocyte protection in CKD patients.

\section{Conclusion}

This study presented a novel approach using WGCNA to explore gene changes during the CKD process, especially when podocyte damage occurs. According to the network constructed by WGCNA, 12 modules were identified, and four were selected for detailed analysis. The green and red modules were identified as podocyte injury-associated modules, which may provide new insights into pathways and genes underlying CKD and podocyte damage. Meanwhile, as a hub gene of the green module, MAGI2 was associated with CKD progression, with its loss promoting podocyte cytoskeletal rearrangement. Overall, WGCNA is an efficient approach to systems biology. By using this procedure, the key node gene MAGI2 was identified, and found to be involved in the regulation of cytoskeletal rearrangement in podocytes, with its loss predisposing to proteinuria and CKD.

\section{Acknowledgements}

This work was supported by the National Natural Science Foundation of China (Grant No. 81600549, to Wan-Peng Wang), the natural science research project of Huai'an city (Grant No. HAB201737, to Wan-peng Wang); National Natural Science Foundation of China (Grant No. 81700586, to Jian-Xiao Shen); the Foundation Research Project of Jiangsu Province (The Natural Science Fund No. BK20160707 to Zhi Zuo); Shanghai Science Health Bureau(No. 20154Y0003, to Xing-hua Shao); Chinese medical association special foundation for medical science(No.15020110599 to Xing-hua Shao). All experiments of this study were authorized by the Ethics Committee of LianShui County People's Hospital.

\section{Disclosure Statement}

The authors declare that they have no competing interests. 


\section{Cellular Physiology Cell Physiol Biochem 2018;51:244-261 \begin{tabular}{ll|l} 
and Biochemistry & $\begin{array}{l}\text { DOI: 10.1159/000495205 } \\
\text { Published onlIne: 16 November } 2018\end{array}$ & $\begin{array}{l}\text { (c) } 2018 \text { The Author(s). Published by S. Karger AG, Basel } \\
\text { www.karger.com/cpb }\end{array}$
\end{tabular}

\section{References}

1 Eckardt KU, Coresh J, Devuyst O, Johnson RJ, Kottgen A, Levey AS, Levin A: Evolving importance of kidney disease: from subspecialty to global health burden. Lancet 2013;382:158-169.

-2 Cechova S, Dong F, Chan F, Kelley MJ, Ruiz P, Le TH: MYH9 E1841K Mutation Augments Proteinuria and Podocyte Injury and Migration. J Am Soc Nephrol 2018;29:155-167.

3 Liu M, Liang K, Zhen J, Zhou M, Wang X, Wang Z, Wei X, Zhang Y, Sun Y, Zhou Z, Su H, Zhang C, Li N, Gao C, Peng J, Yi F: Sirt6 deficiency exacerbates podocyte injury and proteinuria through targeting Notch signaling. Nat Commun 2017;8:413.

-4 Yamamoto-Nonaka K, Koike M, Asanuma K, Takagi M, Oliva Trejo JA, Seki T, Hidaka T, Ichimura K, Sakai T, Tada N, Ueno T, Uchiyama Y, Tomino Y: Cathepsin D in Podocytes Is Important in the Pathogenesis of Proteinuria and CKD. J Am Soc Nephrol 2016;27:2685-2700.

-5 Kriz W, Lemley KV: A potential role for mechanical forces in the detachment of podocytes and the progression of CKD. J Am Soc Nephrol 2015;26:258-269.

6 Langfelder P, Horvath S: WGCNA: an R package for weighted correlation network analysis. BMC Bioinformatics 2008;9:559.

7 Liu P, Lassen E, Nair V, Berthier CC, Suguro M, Sihlbom C, Kretzler M, Betsholtz C, Haraldsson B, Ju W, Ebefors K, Nystrom J: Transcriptomic and Proteomic Profiling Provides Insight into Mesangial Cell Function in IgA Nephropathy. J Am Soc Nephrol 2017;28:2961-2972.

-8 Ju W, Greene CS, Eichinger F, Nair V, Hodgin JB, Bitzer M, Lee YS, Zhu Q, Kehata M, Li M, Jiang S, Rastaldi MP, Cohen CD, Troyanskaya OG, Kretzler M: Defining cell-type specificity at the transcriptional level in human disease. Genome Res 2013;23:1862-1873.

-9 Huang da W, Sherman BT, Lempicki RA: Systematic and integrative analysis of large gene lists using DAVID bioinformatics resources. Nat Protoc 2009;4:44-57.

10 Gautier L, Cope L, Bolstad BM, Irizarry RA: affy--analysis of Affymetrix GeneChip data at the probe level. Bioinformatics 2004;20:307-315.

11 Kupfer P, Guthke R, Pohlers D, Huber R, Koczan D, Kinne RW: Batch correction of microarray data substantially improves the identification of genes differentially expressed in rheumatoid arthritis and osteoarthritis. BMC Med Genomics 2012;5:23.

12 Lue HW, Yang X, Wang R, Qian W, Xu RZ, Lyles R, Osunkoya AO, Zhou BP, Vessella RL, Zayzafoon M, Liu ZR, Zhau HE, Chung LW: LIV-1 promotes prostate cancer epithelial-to-mesenchymal transition and metastasis through HB-EGF shedding and EGFR-mediated ERK signaling. PLoS One 2011;6:e27720.

13 Jiang J, Sun X, Wu W, Li L, Wu H, Zhang L, Yu G, Li Y: Construction and application of a co-expression network in Mycobacterium tuberculosis. Sci Rep 2016;6:28422.

$>14$ Miller JA, Cai C, Langfelder P, Geschwind DH, Kurian SM, Salomon DR, Horvath S: Strategies for aggregating gene expression data: the collapseRows R function. BMC Bioinformatics 2011;12:322.

-15 Huang DW, Sherman BT, Tan Q Collins JR, Alvord WG, Roayaei J, Stephens R, Baseler MW, Lane HC, Lempicki RA: The DAVID Gene Functional Classification Tool: a novel biological module-centric algorithm to functionally analyze large gene lists. Genome Biol 2007;8:R183.

-16 Mundel P, Reiser J, Zuniga Mejia Borja A, Pavenstadt H, Davidson GR, Kriz W, Zeller R: Rearrangements of the cytoskeleton and cell contacts induce process formation during differentiation of conditionally immortalized mouse podocyte cell lines. Exp Cell Res 1997;236:248-258.

$\checkmark 17$ Zhang B, Horvath S: A general framework for weighted gene co-expression network analysis. Stat Appl Genet Mol Biol 2005;4:Article17.

-18 Yamashita M, Yoshida T, Hayashi M: Podocyte NF-kappaB is dispensable for the pathogenesis of renal ischemia-reperfusion injury. Physiol Rep 2016;4:pii:e12912.

19 Jiang Y, Wang W, Liu ZY, Xie Y, Qian Y, Cai XN: Overexpression of miR-130a-3p/301a-3p attenuates high glucose-induced MPC5 podocyte dysfunction through suppression of TNF-alpha signaling. Exp Ther Med 2018;15:1021-1028.

-20 Saurus P, Kuusela S, Lehtonen E, Hyvonen ME, Ristola M, Fogarty CL, Tienari J, Lassenius MI, Forsblom C, Lehto M, Saleem MA, Groop PH, Holthofer H, Lehtonen S: Podocyte apoptosis is prevented by blocking the Toll-like receptor pathway. Cell Death Dis 2015;6:e1752. 


\section{Cellular Physiology Cell Physiol Biochem 2018;51:244-261 and Biochemistry \begin{tabular}{l|l} 
DOI: 10.1159/000495205 & (c) 2018 The Author(s). Published by S. Karger AG, Basel
\end{tabular}

21 Abais JM, Xia M, Li G, Chen Y, Conley SM, Gehr TW, Boini KM, Li PL: Nod-like receptor protein 3 (NLRP3) inflammasome activation and podocyte injury via thioredoxin-interacting protein (TXNIP) during hyperhomocysteinemia. J Biol Chem 2014;289:27159-27168.

22 Dange RB, Agarwal D, Teruyama R, Francis J: Toll-like receptor 4 inhibition within the paraventricular nucleus attenuates blood pressure and inflammatory response in a genetic model of hypertension. J Neuroinflammation 2015;12:31.

-23 Gross V, Tank J, Partke HJ, Plehm R, Diedrich A, da Costa Goncalves AC, Luft FC, Jordan J: Cardiovascular autonomic regulation in Non-Obese Diabetic (NOD) mice. Auton Neurosci 2008;138:108-113.

-24 Hayakawa K, Nakajima S, Hiramatsu N, Okamura M, Huang T, Saito Y, Tagawa Y, Tamai M, Takahashi S, Yao J, Kitamura M: ER stress depresses NF-kappaB activation in mesangial cells through preferential induction of C/EBP beta. J Am Soc Nephrol 2010;21:73-81.

25 Zhang H, Li L, Wang Y, Dong F, Chen X, Liu F, Xu D, Yi F, Kapron CM, Liu J: NF-kappaB signaling maintains the survival of cadmium-exposed human renal glomerular endothelial cells. Int J Mol Med 2016;38:417-422.

26 Yang Y, Han L, Yuan Y, Li J, Hei N, Liang H: Gene co-expression network analysis reveals common systemlevel properties of prognostic genes across cancer types. Nat Commun 2014;5:3231.

27 Li S, Li B, Zheng Y, Li M, Shi L, Pu X: Exploring functions of long noncoding RNAs across multiple cancers through co-expression network. Sci Rep 2017;7:754.

28 Colas C, Pajor AM, Schlessinger A: Structure-Based Identification of Inhibitors for the SLC13 Family of $\mathrm{Na}(+) /$ Dicarboxylate Cotransporters. Biochemistry 2015;54:4900-4908.

29 Zhunussova A, Sen B, Friedman L, Tuleukhanov S, Brooks AD, Sensenig R, Orynbayeva Z: Tumor microenvironment promotes dicarboxylic acid carrier-mediated transport of succinate to fuel prostate cancer mitochondria. Am J Cancer Res 2015;5:1665-1679.

30 Nigam SK: The SLC22 Transporter Family: A Paradigm for the Impact of Drug Transporters on Metabolic Pathways, Signaling, and Disease. Annu Rev Pharmacol Toxicol 2018;58:663-687.

-31 Kim JI, Noh MR, Kim KY, Jang HS, Kim HY, Park KM: Methionine sulfoxide reductase A deficiency exacerbates progression of kidney fibrosis induced by unilateral ureteral obstruction. Free Radic Biol Med 2015;89:201-208.

-32 Nair S, Lee YH, Rousseau E, Cam M, Tataranni PA, Baier LJ, Bogardus C, Permana PA: Increased expression of inflammation-related genes in cultured preadipocytes/stromal vascular cells from obese compared with non-obese Pima Indians. Diabetologia 2005;48:1784-1788.

33 Lindenmeyer MT, Eichinger F, Sen K, Anders HJ, Edenhofer I, Mattinzoli D, Kretzler M, Rastaldi MP, Cohen CD: Systematic analysis of a novel human renal glomerulus-enriched gene expression dataset. PLoS One 2010;5:e11545.

34 Kim JH, Mukherjee A, Madhavan SM, Konieczkowski M, Sedor JR: WT1-interacting protein (Wtip) regulates podocyte phenotype by cell-cell and cell-matrix contact reorganization. Am J Physiol Renal Physiol 2012;302:F103-115.

-35 Warejko JK, Tan W, Daga A, Schapiro D, Lawson JA, Shril S, Lovric S, Ashraf S, Rao J, Hermle T, Jobst-Schwan T, Widmeier E, Majmundar AJ, Schneider R, Gee HY, Schmidt JM, Vivante A, van der Ven AT, Ityel H, Chen J et al.: Whole Exome Sequencing of Patients with Steroid-Resistant Nephrotic Syndrome. Clin J Am Soc Nephrol 2018;13:53-62.

-36 Kato T, Mizuguchi N, Ito A: Characteristics of podocyte injury in malignant hypertensive nephropathy of rats (MSHRSP/Kpo strain). Biomed Res 2015;36:313-321.

-37 Wen CP, Cheng TY, Tsai MK, Chang YC, Chan HT, Tsai SP, Chiang PH, Hsu CC, Sung PK, Hsu YH, Wen SF: Allcause mortality attributable to chronic kidney disease: a prospective cohort study based on 462293 adults in Taiwan. Lancet 2008;371:2173-2182.

-38 Murphy D, McCulloch CE, Lin F, Banerjee T, Bragg-Gresham JL, Eberhardt MS, Morgenstern H, Pavkov ME, Saran R, Powe NR, Hsu CY, Centers for Disease C, Prevention Chronic Kidney Disease Surveillance T: Trends in Prevalence of Chronic Kidney Disease in the United States. Ann Intern Med 2016;165:473-481.

-39 Hallan SI, Coresh J, Astor BC, Asberg A, Powe NR, Romundstad S, Hallan HA, Lydersen S, Holmen J: International comparison of the relationship of chronic kidney disease prevalence and ESRD risk. J Am Soc Nephrol 2006;17:2275-2284.

-40 Wu J, Jia J, Li Z, Pan H, Wang A, Guo X, Wu S, Zhao X: Association of estimated glomerular filtration rate and proteinuria with all-cause mortality in community-based population in China: A Result from Kailuan Study. Sci Rep 2018;8:2157. 


\section{Cellular Physiology Cell Physiol Biochem 2018;51:244-261

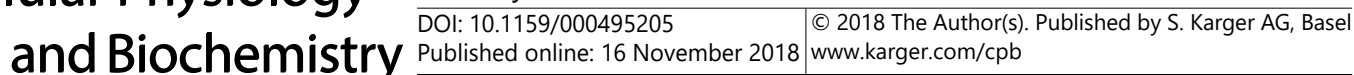 \\ Zuo et al.: Loss of MAGI2 Promoted Podocyte Damage}

41 Chen CH, Wu HY, Wang CL, Yang FJ, Wu PC, Hung SC, Kan WC, Yang CW, Chiang CK, Huang JW, Hung KY: Proteinuria as a Therapeutic Target in Advanced Chronic Kidney Disease: a Retrospective Multicenter Cohort Study. Sci Rep 2016;6:26539.

42 Shankland SJ: The podocyte's response to injury: role in proteinuria and glomerulosclerosis. Kidney Int 2006;69:2131-2147.

-43 Sun L, Zhu J, Wu M, Sun H, Zhou C, Fu L, Xu C, Mei C: Inhibition of MiR-199a-5p reduced cell proliferation in autosomal dominant polycystic kidney disease through targeting CDKN1C. Med Sci Monit 2015;21:195200.

-44 Piersigilli F, Auriti C, Mondi V, Francalanci P, Salvatori G, Danhaive O: Decreased CDKN1C Expression in Congenital Alveolar Rhabdomyosarcoma Associated with Beckwith-Wiedemann Syndrome. Indian J Pediatr 2016;83:1476-1478.

45 Wang S, Kim JH, Moon KC, Hong HK, Lee HS: Cell-cycle mechanisms involved in podocyte proliferation in cellular lesion of focal segmental glomerulosclerosis. Am J Kidney Dis 2004;43:19-27.

46 Hatta Y, Nakamura A, Hara S, Kamijo T, Iwata J, Hamajima T, Abe M, Okada M, Ushio M, Tsuyuki K, Tajima T: Clinical and molecular analysis of six Japanese patients with a renal form of pseudohypoaldosteronism type 1. Endocr J 2013;60:299-304.

47 Christakoudi S, Runglall M, Mobillo P, Rebollo-Mesa I, Tsui TL, Nova-Lamperti E, Norris S, Kamra Y, Hilton R, Bhandari S, Baker R, Berglund D, Carr S, Game D, Griffin S, Kalra PA, Lewis R, Mark PB, Marks SD et al.: Steroid regulation: An overlooked aspect of tolerance and chronic rejection in kidney transplantation. Mol Cell Endocrinol 2018;10.1016/j.mce.2018.01.021.

-48 Steubl D, Kumar SV, Tato M, Mulay SR, Larsson A, Lind L, Riserus U, Renders L, Heemann U, Carlsson AC, Arnlov J, Anders HJ: Circulating cathepsin-S levels correlate with GFR decline and sTNFR1 and sTNFR2 levels in mice and humans. Sci Rep 2017;7:43538.

-49 Sena BF, Figueiredo JL, Aikawa E: Cathepsin S As an Inhibitor of Cardiovascular Inflammation and Calcification in Chronic Kidney Disease. Front Cardiovasc Med 2017;4:88.

50 Stuart JM, Segal E, Koller D, Kim SK: A gene-coexpression network for global discovery of conserved genetic modules. Science 2003;302:249-255.

51 Signal B, Gloss BS, Dinger ME: Computational Approaches for Functional Prediction and Characterisation of Long Noncoding RNAs. Trends Genet 2016;32:620-637.

52 Li J, Li YX, Li YY: Differential Regulatory Analysis Based on Coexpression Network in Cancer Research. Biomed Res Int 2016;2016:4241293.

53 Debiec H, Nauta J, Coulet F, van der Burg M, Guigonis V, Schurmans T, de Heer E, Soubrier F, Janssen F, Ronco P: Role of truncating mutations in MME gene in fetomaternal alloimmunisation and antenatal glomerulopathies. Lancet 2004;364:1252-1259.

54 Cunningham PN, Quigg RJ: Contrasting roles of complement activation and its regulation in membranous nephropathy. J Am Soc Nephrol 2005;16:1214-1222.

55 Sugano Y, Lindenmeyer MT, Auberger I, Ziegler U, Segerer S, Cohen CD, Neuhauss SC, Loffing J: The RhoGTPase binding protein IQGAP2 is required for the glomerular filtration barrier. Kidney Int 2015;88:10471056.

56 Ihara K, Asanuma K, Fukuda T, Ohwada S, Yoshida M, Nishimori K: MAGI-2 is critical for the formation and maintenance of the glomerular filtration barrier in mouse kidney. Am J Pathol 2014;184:2699-2708.

57 Dong L, Pietsch S, Tan Z, Perner B, Sierig R, Kruspe D, Groth M, Witzgall R, Grone HJ, Platzer M, Englert C: Integration of Cistromic and Transcriptomic Analyses Identifies Nphs2, Mafb, and Magi2 as Wilms' Tumor 1 Target Genes in Podocyte Differentiation and Maintenance. J Am Soc Nephrol 2015;26:2118-2128.

58 Balbas MD, Burgess MR, Murali R, Wongvipat J, Skaggs BJ, Mundel P, Weins A, Sawyers CL: MAGI-2 scaffold protein is critical for kidney barrier function. Proc Natl Acad Sci U S A 2014;111:14876-14881.

59 Kim BS, Lee JG, Cho Y, Song SH, Huh KH, Kim MS, Kim YS: Reduction of Slit Diaphragm-associated Molecules by Sirolimus: Is it Enough to Induce Proteinuria? Transplant Proc 2017;49:1165-1169.

60 Yuan Y, Huang S, Wang W, Wang Y, Zhang P, Zhu C, Ding G, Liu B, Yang T, Zhang A: Activation of peroxisome proliferator-activated receptor-gamma coactivator 1alpha ameliorates mitochondrial dysfunction and protects podocytes from aldosterone-induced injury. Kidney Int 2012;82:771-789.

61 Dong N, Meng L, Xue R, Yu M, Zhao Z, Liu X: Adrenomedullin ameliorates podocyte injury induced by puromycin aminonucleoside in vitro and in vivo through modulation of Rho GTPases. Int Urol Nephrol 2017;49:1489-1506. 